\title{
La enfermedad de Parkinson: un enfoque interdisciplinario ${ }^{1}$
}

\author{
Luis Aguilar \\ Walter Riofrío \\ Universidad Peruana Cayetano Heredia \\ Ricardo Braun \\ Universidad de Lima \\ Rocío Callupe \\ Pontificia Universidad Católica del Perú
}

Recibido: 21 de marzo del 2016 / Aceptado: 25 de abril del 2016

En este articulo resumimos la experiencia de una investigación interdisciplinaria sobre la enfermedad de Parkinson, que reunió a un equipo proveniente de la neurociencia, biología y ciencia de la complejidad, ingeniería biomédica y filosofía de la mente. Se sugiere que, dados el aumento de la concentración y su presencia en los núcleos reportados, neuropéptido $Y$ (NPY) podría ser un indicador confiable de esta enfermedad. Si la red neuronal prepara al cerebro para la actividad consciente y entonces juega un rol en la experiencia consciente. En los aspectos psicológico y social, la pérdida de la identidad no solo se da en términos genéticos y fisiológicos, sino que, además, tenemos que tomar en cuenta la interacción con el ambiente. Por eso es necesario entender la enfermedad de Parkinson como una enfermedad a diferentes niveles, desde el molecular hasta el social.

enfermedad de Parkinson / neuropéptido Y / interdisciplinariedad / enfermedad multinivel

\section{Parkinson's Disease: an Interdisciplinary Approach}

In this paper we summarize an interdisciplinary study on Parkinson's disease. Members of the team were drawn from neuroscience, biology and complex sciences, biomedical engineering, and philosophy of mind. Due to its concentration and presence on reported nuclei, we suggest that neuropeptide Y may be considered a reliable indicator of this disease. If the neuro network participates on conscious experience, it would certainly play a role on consciousness. Moreover, on psychological and social levels, Parkinson's disease is involved in the loss of identity which includes not only genetic and physiological factors, but environment interaction as well. Therefore, Parkinson's disease should be considered a multi-level disease, ranging from the molecular to the social.

Parkinson's disease / neuropeptide Y / interdisciplinary studies / multi-level disease

Correo electrónico: walter.riofrio.r@upch.pe

1 Agradecemos al Consorcio de Universidades (www.consorcio.edu.pe) por el apoyo recibido para la realización del proyecto y la autorización para publicar los resultados. 
Las reflexiones que a continuación consignamos son el resultado del trabajo conjunto de cuatro académicos y el equipo que lo acompaña en una investigación interdisciplinar encomendada por el Consorcio de Universidades. El Consorcio agrupa a las cuatro universidades privadas con mayor prestigio en el Perú y en el año 2014 promovió la formación de equipos interdisciplinarios para estudiar un problema, pero desde un enfoque interdisciplinario e interuniversitario. Nuestro grupo, en concurso abierto, obtuvo el tercer puesto en la convocatoria con la propuesta "Cambios de expresión del neuropéptido $\mathrm{Y}$ en el sistema nervioso central de un modelo animal con Parkinson".

Esta propuesta, como era de esperar, requería enfrentarla con la visión de diferentes disciplinas, lo que constituía un reto de investigación por dos motivos: 1) conceptualización de un problema a nivel interdisciplinario y 2) alejamiento de la zona de confort disciplinario al que estamos acostumbrados.

Veamos algunas reflexiones conjuntas.

\section{LA EXPERIENCIA MULTIDISCIPLINAR EN LA CIENCIA}

El presente trabajo es el resultado de la iniciativa del Consorcio de Universidades para promover el trabajo interdisciplinario e interuniversitario. Esta iniciativa parte de la convicción de que se requiere empezar a pensar, investigar y trabajar con el concurso de especialistas de diferentes áreas $\mathrm{y}$, asunto adicional, interesados en abordar un mismo tema de investigación. Esto no es fácil.

La investigación interdisciplinaria no es nueva. En efecto, una de las áreas que más ha cosechado frutos de los esfuerzos colectivos es la ciencia cognitiva. Y, por ello, nos permitimos comentar sobre esta posible nueva ciencia como un ejemplo de un trabajo multidisciplinar exitoso. Este ejemplo nos ha permitido reflexionar sobre nuestro propio trabajo en el presente proyecto.

La ciencia cognitiva es llamada por algunos la "nueva ciencia de la mente" (Gardner, 1985). Con el fracaso de la visión reduccionista del conductismo, y con el surgimiento de la ingeniería de la inteligencia artificial, se encontró una forma de modelar mejor la inteligencia humana a partir del modelo computacional, aun cuando el modelo siguiente suponga la refutación del que le dio origen.

Este modelo provocó una serie de preguntas que requería la colaboración de especialistas de diferentes áreas, puesto que se abordaban cuestiones que no podían ser resueltas por una sola disciplina. Así, el esfuerzo conjunto se abocó a tratar problemas acerca de la adquisición, almacenamiento y funcionamiento de la inteligencia artificial. $\mathrm{Y}$ encontraron que científicos de la psicología, ingeniería, computación, lingüística y neurociencia podían ayudar en esta empresa compleja. También se pensó que era importante incorporar a filósofos, por su tradicional visión integradora de diferentes áreas del conocimiento, y, por lo tanto, producir una visión sinóptica del trabajo. En la 
actualidad, se incorpora, además, a los antropólogos, para tratar de entender el aspecto cultural en un modelo, hasta el momento, computacional.

¿Podríamos decir, como ejemplo a seguir, que los departamentos de ciencia cognitiva trabajan en forma interdisciplinaria? Esta pregunta es difícil de contestar si con esto nos referimos a la forma del trabajo científico cotidiano. De hecho, algunos autores que trabajan como miembros de departamentos de ciencia cognitiva no están de acuerdo con catalogarla como tal. John Searle, de la Universidad de California (Berkeley), lo dice elocuentemente:

No estoy seguro que hay tal cosa como la ciencia cognitiva. Pero es frecuentemente útil juntar a personas con intereses comunes y ponerlos a trabajar juntos. No tomo la organización por departamentos universitarios muy en serio: ¿es usted realmente un filósofo, o un lingüista, o un historiador, o un psicólogo, o un científico cognitivo? Esas preguntas no son realmente muy interesantes, y yo creo que esas son preocupaciones para los decanos universitarios - muy útiles para los presupuestos. (1995, pp. 203-204)

Este comentario resulta iluminador cuando reflexionamos acerca de la importancia (o no) de las nomenclaturas o denominaciones que tienen los estudios interdisciplinarios para luego abandonar lo que parece esencial: el interés común por un tema problemático. Pero además revela la diversidad de interpretación que puede originar un concepto como el de interdisciplinariedad. Este concepto puede ser vago, pero, al mismo tiempo, lo percibimos como uno relevante y digno de tomarse seriamente.

Parte de la importancia, creemos, radica en que nos damos cuenta, intuitiva o reflexivamente, de que algunos problemas sobrepasan la capacidad explicativa de una sola disciplina y requieren de la colaboración de personas formadas en diferentes campos. Sin embargo, el interés común puede o no traer diferentes puntos de vista.

Se puede pensar en un trabajo interdisciplinario al menos en dos formas: unos investigadores pueden concebir que el trabajo consiste en aprender los métodos y problemas de otros campos para mejorar su propia práctica; otros pueden utilizar los hallazgos y resultados de otras especialidades para aplicarlos al propio, continuando con los estilos y el mismo campo de trabajo en el que fueron entrenados. De allí que nuestro equipo haya tratado de esclarecer, en la medida de la dificultad señalada, esta primera encrucijada conceptual.

Hace una década, la Academia Nacional de las Ciencias de los Estados Unidos de América, publicó un reporte bajo el título de "Facilitando la investigación interdisciplinaria" [Facilitating Interdisciplinary Research], en el que señalaba la importancia capital de investigar algunos problemas desde la perspectiva de, por lo menos, más de una disciplina, puesto que algunos problemas eran demasiado complejos para ser abordados solitariamente por una disciplina. Así señalaba, optimista, el documento: 
La investigación interdisciplinaria (IID) puede ser una de las búsquedas humanas más productivas e inspiradoras - una que proporcione un formato para conversaciones y conexiones que conduzcan a un nuevo conocimiento. Como una forma de descubrimiento y educación, ya ha proporcionado mucho y promete aún más -un entorno sostenible, unas vidas más saludables y prósperas, nuevos descubrimientos y tecnologías que inspiren a las mentes jóvenes, y una comprensión más profunda de nuestro lugar en el espacio y en el tiempo. Y a pesar de los beneficios aparentes de la IID, los investigadores que la buscan se enfrentan a obstáculos desalentadores y a pocos incentivos. (National Academy of Sciences, 2005, p. 1)

S. Bennett, que ocupó la presidencia de la Asociación Americana de Psicología (APA), señalaba en 2012 que, aun cuando encontraba que había un interés por la investigación interdisciplinaria, los obstáculos provenían de una cultura tradicional que parecía desafiar esta motivación. Enumeraba algunas características: 1) la organización de las universidades por departamentos, que suelen ser espacios cerrados, que promueven poco la ciencia interdisciplinaria; 2) los comités que recomiendan la renovación o promoción de los docentes recompensan la autoría individual de artículos o libros, cuando usualmente los trabajos interdisciplinarios involucran típicamente la autoría de múltiples autores, en algunos casos, de muchos; 3) las revistas científicas actuales no proporcionan una plataforma para la publicación de trabajos interdisciplinarios; 4) los científicos que asesoran a las nuevas generaciones no tienen las herramientas o el interés para encaminarse a las nuevas áreas de la investigación interdisciplinaria (Bennett, 2012).

Sumada a las posibles barreras administrativas, señalaba que, en los equipos interdisciplinarios, los científicos de las tradicionalmente (y posiblemente mal) llamadas "ciencias duras" solían tener actitudes de desconfianza particularmente con las ciencias humanas, en particular con la psicología, que consideraban algo menos que una buena ciencia. Sin embargo, de acuerdo a la propia experiencia de Bennett, los otros científicos empiezan a apreciar el aporte de la psicología, pero solo después de demostrar su valor como una ciencia propiamente dicha.

Nuestra intención, por supuesto, no es presentar un futuro poco promisorio, sino, al contrario, al señalar algunas de las dificultades, motivar la búsqueda para superar los obstáculos mencionados y aquellos que son más propios de nuestra propia realidad peruana, y proponer posteriormente soluciones en la creación de esta nueva cultura, que si bien goza de la aceptación inicial -o llamada de emergencia, como quiera interpretarse-, todavía enfrenta una lentitud que esperamos no continúe. Nuestra experiencia en esta investigación de la enfermedad de Parkinson, entre otros esfuerzos similares, demuestra que es posible dar los primeros pasos. 


\section{EL ENFOQUE NO REDUCCIONISTA}

\section{DEL PROYECTO}

Hemos tomado una posición más cercana a la primera de las dos alternativas. Es decir, hemos preferido aprender y tratar de poner en práctica las formas de investigación que traemos de nuestra diferente formación. Pero, y aquí está quizás el escollo más profundo, el conjunto de suposiciones y concepciones suele ser el más complejo de compartir, y este trabajo resume esta problemática en la dinámica incipiente del trabajo colectivo.

El punto de partida compartido ha sido el rechazo al reduccionismo tanto en la metodología como en la discusión de los resultados de la investigación metafísica. Como se explicará más adelante, la visión que tenemos es de la realidad plural en sentido ontológico, y que esta se expresa en diferentes descripciones que se expondrán en los capítulos siguientes. El haber alcanzado o no en nuestro trabajo individual esta visión compartida es algo que se podrá juzgar eventualmente. Sin embargo, es importante recalcar la dificultad y el proceso para llegar a un punto de convergencia que, si bien desde el principio fue aceptado, en el trabajo práctico no siempre se podía encontrar. Y por ello consideramos que este trabajo no solo forma parte de un compromiso cumplido, sino que constituye un proceso de maduración como investigadores científicos de una nueva generación.

El trabajo encomendado por el Consorcio de Universidades tiene una característica institucional que se debe mencionar. Los miembros del equipo de esta investigación son profesores que pertenecen a tres de las universidades del Consorcio. Este representa una visión compartida de universidad que ha permitido el fácil acercamiento y colaboración entre los miembros: pertenecemos a universidades privadas sin fines de lucro, con una reconocida trayectoria nacional y creciente visibilidad internacional; universidades que apoyan evidentemente la investigación científica; universidades que confían en sus investigadores. Esos elementos han resultado, en la práctica, en un motivador extrínseco para provocar un adecuado intercambio formal e informal en un clima idóneo. Queda claro que un ambiente así permite una influencia positiva y provoca una motivación para poder ampliar la perspectiva individual. El trabajo interdisciplinar parece exigir este tipo de requisito: un adecuado rol institucional.

Nos preguntamos, al terminar el trabajo, y por lo tanto redactar la introducción, si esta visión institucional tendría un rol en el futuro de investigaciones similares o diferentes. Nos referimos al rol institucional que toma el Consorcio acerca de la consolidación de diseños de programas que confieran al trabajo interdisciplinario una realidad continua. Tal diseño supone cambiar nuestros modos de organizar la forma de adquisición y trasmisión del conocimiento, partiendo de un nuevo modelo basado en la interdisciplinariedad.

La creación de carreras o departamentos interdisciplinarios es una alternativa que supondría, eventualmente, la formación de nuevas especialidades, con 
investigadores y estudiantes entrenados para pensar y actuar de esta forma. Para volver al ejemplo propuesto, numerosas universidades del mundo cuentan actualmente con departamentos de ciencia cognitiva, y forman estudiantes con un currículonovedoso, pero interdisciplinario.

Es prematuro evaluar los resultados, puesto que se esperará un grupo de segunda generación que redefina los problemas que, se supone, la primera generación está abordando con este modelo.

Sin embargo, y como esta discusión pretende mostrar también, es posible que el modelo multidisciplinario no pueda llevarse a cabo en la realidad. El conocimiento actual es tan vasto que pareciera una quimera formar estudiantes en varias especialidades a nivel de expertos. Se temería que fueran conocedores de muchas cosas, pero expertos en nada. Tómese en cuenta la dificultad que atraviesan docentes, investigadores y estudiantes para dominar un solo campo de estudio, dada la sobreespecialización a la que se ven desafiados. Esta segunda opción no tiene que ser vista de forma pesimista como un fracaso en los intentos de entender los problemas en forma interdisciplinaria, puesto que, dado el clima favorable para la comprensión interdisciplinaria de algunos problemas, los nuevos currículos podrán empezar de la forma tradicional -de lo especializado a lo multidisciplinario- para que, en el futuro, la dirección sea la inversa: partir de la visión multidisciplinaria de la disciplina como un núcleo propedéutico para dar lugar a un campo especializado. El punto de partida multidisciplinario permitiría entender los problemas y objetivos tal como lo plantean las diversas disciplinas para dar lugar, en una segunda o tercera generación, a una nueva forma de especialización.

Es evidente que nuestra visión es altamente especulativa; sin embargo, es menester expresarla como resultado de nuestras numerosas deliberaciones acerca y fuera del proyecto mismo, en cuanto, reiteramos, miembros de la comunidad de investigadores del Consorcio.

\section{LA PLURALIDAD DE LA REALIDAD}

La concepción pluralista de la realidad se define, actualmente, en términos asociados a otro concepto que presentamos para elaborar nuestra visión: emergentismo.

El término emergencia es utilizado en diferentes sentidos, pero nos concentraremos no tanto en el uso no popular, sino más bien en el vocabulario empleado en el ambiente académico contemporáneo. El concepto de emergentismo es usado, generalmente, en las doctrinas de orientación no-reduccionistas tanto de la biología como de la psicología y otras disciplinas sociales.

Desde una perspectiva metafísica, una postura emergentista concibe que la realidad existe y se presenta en forma plural, de ahí que, en primer lugar, se pueda afirmar que el emergentismo es una forma de pluralismo (Bunge, 1977). Sin embargo, el pluralismo que da sentido al emergentismo debe admitir que existen diferentes niveles de organización que son ontológicamente de igual valor. 
En segundo lugar, el emergentismo sostiene que, en cada nivel superior de organización, aparecen (o emergen) nuevas e irreductibles propiedades que no están presentes en el nivel inferior. Estas propiedades serían "emergentes", predicadas de un todo organizado, y no de sus partes o componentes.

Otra forma de presentar la tesis emergentista es sosteniendo que las propiedades de mayor nivel son propiedades de segundo orden, que son el resultado (o emergen) de las de primer orden.

Desde una perspectiva epistemológica, cualquier doctrina emergentista supone que el conocimiento de las propiedades emergentes no puede ser derivado del conocimiento de las propiedades de las partes que componen el ente emergente, y las leyes que se puedan formular en el nivel emergente no son deducibles de las que se obtienen en los niveles inferiores.

Tomemos la esquizofrenia como un ejemplo. Es una enfermedad que tiene factores biológicos, sociales y ambientales que sugieren que existe en diferentes niveles de organización. Así, una perspectiva de nivel múltiple de la esquizofrenia evita un miope enfoque único de esta compleja enfermedad que no solo produciría una versión incompleta, sino posiblemente falsa de la enfermedad.

Los casos descritos pueden proporcionarnos un marco de referencia para la unidad que tenemos a nivel cerebral. La diversidad de la realidad, dada la evolución cósmica, requiere una apropiada construcción mental de la organización jerárquica de los fenómenos para aprehender las diferentes propiedades de los objetos (sean los átomos, la tierra, las células, los organismos, los grupos sociales, etc.). Inevitablemente, las diferencias entre propiedades requieren un enfoque pluralista en la explicación, no solo de orden epistemológico, sino también ontológico.

El proyecto reduccionista presupone una explicación ontológica monista. Pero esta presuposición no toma en cuenta solo la teoría científica, sino la práctica científica. Consideremos nuevamente la diferencia entre la física relativista y la newtoniana. Una habla de espacio curvo, la otra de fuerzas gravitatorias. Preguntar cuál metafísica es la correcta sería simplemente no entender el punto de la explicación física. Aquí hay dos ontologías. Cada una proporciona una intuición en la explicación de los fenómenos. Este ejemplo es análogo a muchos que encontramos en las diferentes ciencias. ¿Por qué deberíamos esperar que todo fuera explicado con una misma ontología? La historia reciente de la física nos demuestra la importancia de reconocer una metafísica pluralista. La controversia acerca de la mecánica cuántica nos proporciona una luz para darnos cuenta del mundo en que vivimos. Y el mundo no parece estar de acuerdo con las expectativas de algunos reduccionistas. Así lo señala McMullin:

¿Por qué hemos pretendido que la ontología del micromundo sea igual al del macromundo? La tercera regla de Newton para filosofar (que decretaba que el macromundo debiera ser semejante al micromundo en todos sus detalles esenciales) no ha sido más que una esperanza piadosa. (1984, p.13) 
Una metafísica pluralista no es incompatible con la unidad de la ciencia. Al contrario, puesto que no hay una última verdad científica conocida, la integración entre los niveles de las teorías puede demostrar ser una explicación más fructífera y acertada que, de otro modo, se nos presentaría como un mundo de eventos inconexos.

\section{Aplicación del pluralismo \\ a la enfermedad de Parkinson}

Tomando en cuenta todo lo anteriormente mencionado, se puede señalar que la enfermedad de Parkinson se presenta cuando se dañan o mueren un conjunto de neuronas en el cerebro. Aun cuando muchas áreas del cerebro se ven afectadas, los síntomas más comunes son el resultado de la pérdida de neuronas en un área cerca de la base del cerebro conocida como sustancia negra.

El Parkinson es una enfermedad de carácter crónico, progresivo y lento, que afecta a la zona del cerebro encargada del control y coordinación del movimiento, del tono muscular y de la postura.

En esta zona, la sustancia negra, existe un componente químico, la dopamina, compuesto esencial para la regulación de los movimientos, es decir, para que los movimientos se realicen de una forma efectiva y armónica.

Así, en el Parkinson se produce una "degeneración" de la sustancia negra por causas diversas, cuya consecuencia es la disminución de la dopamina. Es por ello que las principales manifestaciones de la enfermedad expresan un control deficiente de los movimientos: temblor, lentitud generalizada (bradicinesia), rigidez y alteraciones de la postura y de la marcha.

Además, la dopamina es el mensajero químico responsable de transmitir señales a partir de la sustancia negra a la siguiente región del cerebro, llamada el cuerpo estriado, para producir movimientos uniformes y deliberados.

La pérdida de dopamina produce patrones anormales de activación nerviosa dentro del cerebro, que causan deterioro del movimiento. Los estudios demuestran que la mayoría de las personas con Parkinson han perdido de un 60 a un 80 por ciento, o más, de las células productoras de dopamina en la sustancia negra en el momento de la aparición de los síntomas, y que también se genera una pérdida de las terminaciones nerviosas que producen el neurotransmisor norepinefrina.

Se sabe que la norepinefrina está estrechamente relacionada con la dopamina. Es el mensajero químico excitatorio principal del sistema nervioso simpático, la parte del sistema nervioso que controla muchas de las funciones automáticas del cuerpo, como el pulso y la presión arterial. La pérdida de norepinefrina puede ayudar a explicar varias de las características no motrices que se ven en la enfermedad de Parkinson, entre ellas, la fatiga y las anomalías relacionadas con la regulación de la presión arterial.

Así, además, se ha postulado que los niveles bajos tanto de dopamina como de norepinefrina estarían relacionados con 
la depresión (un síntoma que presentan muchos pacientes con Parkinson).

Pero, a pesar de que la correlación entre las deficiencias del comportamiento motor y los desniveles de ciertos componentes moleculares parece bastante clara, ¿cómo las variaciones de los niveles de estos compuestos moleculares se correlacionarían con ciertos fenómenos (y patologías) de la conciencia humana? Preguntas de esta clase son las que nos han animado a lo largo de nuestra investigación.

De esta manera, podemos afirmar que el Parkinson es una enfermedad de origen neurológico que involucra varios niveles; cada uno de ellos nos puede mostrar facetas muy específicas del desarrollo y desenvolvimiento de esta enfermedad.

El clima científico actual está enfocado en la biología celular o los genes que las células contienen o activan. Sabemos más acerca de las células que conforman el cerebro, y cómo estas se conectan o modifican sus conexiones. Los recientes avances relacionados con nuestro entendimiento del genoma han tenido efectos en la investigación sobre el cerebro, así como en casi como todos los demás campos. Estos son tiempos emocionantes para la biología molecular, celular y genética. Aun así, el salto hacia la función del cerebro como órgano aún parece insuperable, razón por la cual la psiquiatría seguirá siendo de la manera en la que es ahora por algún tiempo (Herbert, 2015). El problema de una visión mecanicista y reduccionista de las enfermedades mentales es que dichas concepciones no contribuyen a su entendimiento total e integrado. Es por ello que queremos brindar un panorama global, desde el punto de vista psicológico y social de esta enfermedad.

Además, quisiéramos resaltar la importancia de la investigación interdisciplinaria. Para comenzar, una disciplina puede definirse convenientemente como cualquier dominio aislado comparativamente autónomo de la experiencia humana que posee su propia comunidad de expertos. La interdisciplinariedad es mejor vista como el agrupamiento de distintos componentes de dos o más disciplinas. En el discurso académico, la interdisciplinariedad, típicamente, se aplica a cuatro ámbitos: conocimiento, investigación, educación y teoría. La investigación interdisciplinaria combina componentes de dos o más disciplinas en la búsqueda o creación de nuevos conocimientos (Nissani, 1997). Un enfoque interdisciplinario debería llevar a la gente a hacer preguntas y a resolver problemas nunca antes vistos. Pero también puede abordar antiguos problemas, especialmente aquellos que han sido reacios a ceder a enfoques convencionales (Nature, 2015), como sería el caso del tratamiento de la enfermedad de Parkinson, ya que esta y muchas otras enfermedades tienen una repercusión en diversos aspectos de las personas no solo a nivel físico, sino también psicológico y social. Ya que el ser humano abarca muchos aspectos, contextos y realidades a medida que se desarrolla, las enfermedades cambian e interactúan con dichos aspectos; por ello es de suma importancia mantener una perspectiva amplia. Además, la comunicación es crucial. El uso variado del 
lenguaje entre disciplinas podría ser visto como un problema superficial, pero es uno que debe ser resuelto; por el contrario, los malentendidos socavarán las bases del proyecto. Debe haber una jerarquía o una jerarquía percibida. Todo lo involucrado debe tener la confianza de que los colegas de otras disciplinas usen el mismo rigor académico y nivel científico, aun si los métodos en campos rivales parecieran ajenos. Tomará tiempo ver el valor de estos enfoques. Ello requiere de una mentalidad creativa e innovadora (Nature, 2015).

Para poder ejemplificar esta perspectiva interdisciplinaria, supongamos que quisiéremos entender la Guerra Fría. Supongamos, además, que estuviéramos interesados en sondear el conflicto entero, no meramente uno u otro de sus aspectos. Varios años y varios estantes después, podríamos darnos cuenta de que muchos de los expertos no han podido llegar a un retrato autónomo e integrado de este hecho, porque han examinado este tema desde una única perspectiva disciplinaria. En un enfoque integrado, podríamos concluir, surge una gran promesa de alcanzar una mejor comprensión de este tema tan complejo. Sin embargo, en esta instancia particular, podríamos comenzar con historia. En cierto punto, en nuestra ambiciosa tarea, podríamos darnos cuenta de que la historia queda corta, y de que las políticas de ambos rivales, Estados Unidos y Rusia, son un tema importante. Y en otro punto podríamos concluir que las teorías y prácticas del totalitarismo y la democracia deberían entenderse también. Podríamos prolongar este proceso ramificado por un tiempo, hasta que una imagen coherente- mente razonable emerja. Si preserváramos nuestra amplia síntesis, podríamos encarnar un profundo entendimiento que ningún enfoque unidisciplinario podría reunir (Nissani, 1997).

Así como la Guerra Fría podría considerarse un fenómeno complejo, asimismo la enfermedad de Parkinson también lo es; por ende, es de suma importancia poder concebirla desde esta perspectiva interdisciplinaria poder tener un alcance real que nos permita estudiarla en todos sus aspectos, y de tal manera construir un conocimiento fehaciente y verídico de todos los aspectos involucrados, tanto a nivel orgánico como en los niveles más altos de organización de la materia, es decir, a nivel social.

\section{Referencias}

Bennett, S. (2012). Increasing psychology's role in interdisciplinary science. Monitor on Psychology, 43(2), 5.

Bunge, M. (1977). Emergence and the mind. Neuroscience, 2, 501-509.

Gardner, H. (1985). The Mind's New Science: A History of the Cognitive Revolution. New York: Basic Books

Herbert, J. (2015). Craking the Skull Open. Recuperado de https://aeon. co/essays/why-can-t-we-treat-mentalillness-by-fixing-the-brain.

McMullin, E. (1984). A Case for Scientific Realism. En J. Leplin (Ed.), Scientific Realism. Berkeley, CA: University of California Press. 
National Academy of Sciences. (2005). Facilitating Interdisciplinary Research. Washington, D. C.: The National Academies Press.

Nature Publishing Group. (2015). Mind Meld:Interdisciplinary Science Must Breakdown Barriers Between Fields to Build Common Ground. Nature News, 525.

Nissani, M. (1997). Diez aplausos por la interdisciplinariedad. Social Science Journal, 34(2), 201-216.

Searle, J. (1995). The Construction of Social Reality. New York: The Free Press. 
